\title{
Análisis Craneocervical en Sujetos con Respiración Oral y Nasal
}

\author{
Craneocervical Analysis in Subjects With Oral and Nasal Breathing
}

María P. Moya ${ }^{1}$; Sergio Olate ${ }^{2,3,4}$ \& Juan P. Baeza ${ }^{1}$

MOYA, M. P.; OLATE, S. \& BAEZA, J. P. Análisis Craneocervical en Sujetos con Respiración Oral y Nasal. Int. J. Morphol., 37(2):724-729, 2019.

RESUMEN: La influencia de la función respiratoria en el desarrollo de estructuras orofaciales y postura craneocervical ha sido ampliamente discutida. El objetivo del estudio fue comparar valores cefalométricos de la región craneocervical e hioidea en sujetos con respiración nasal y oral. Se incluyeron sujetos de entre 18 y 27 años, de ambos sexos, donde 20 presentaban diagnóstico de respiración oral y 20 no presentaban esta alteración; mediante telerradiografía lateral de cabeza y cuello se realizó análisis cefalométrico craneocervical de Rocabado y aplicación de la técnica de Penning, obteniendo medidas craneocervicales e hioideas, dimensión anterior nasofaríngea y curvatura cervical. Para el análisis estadístico se utilizó la prueba de normalidad Shapiro-Wilk y la prueba T para muestras independientes, considerando un valor de $\mathrm{p}<0,05$ para obtener diferencias significativas; en aquellos parámetros en donde no se presentó distribución normal se aplicó la prueba U de Mann-Whitney. No se encontraron diferencias significativas entre los grupos de estudio y los valores cefalométricos analizados, a excepción de la distancia entre la base del hueso occipital y el arco posterior del atlas ( $\mathrm{p}=0,03$ ). Existen limitadas diferencias cefalométricas entre sujetos con respiración oral y respiración nasal, no asociándose el espacio aéreo nasofaríngeo con las modalidades de respiración estudiadas. Deben ser consideradas condiciones de morfología facial o mandibular, para determinar más adecuadamente la influencia de los parámetros cefalométricos en el diagnóstico del modo respiratorio en estudios futuros.

PALABRAS CLAVE: Respiración, Obstrucción nasal, Cefalometría, Postura, Hueso hioides.

\section{INTRODUCCIÓN}

El trabajo armónico del sistema estomatognático responde a diferentes tareas funcionales, contribuyendo también al control postural de la región cervical. El análisis de la postura corporal mediante anatomía radiográfica ha sido objeto de múltiples estudios desde la primera mitad del siglo XX, permitiendo establecer su relación con aspectos tan diversos como el crecimiento craneofacial (Solow \& Siersbaek-Nielsen, 1992), disfunciones de la articulación temporomandibular (Lee et al., 1995; Rodríguez Romero et al., 2004; Matheus et al., 2009), función respiratoria (Inoko \& Morita, 2009; Svanholt et al., 2009; Chung Leng Muñoz \& Beltri Orta, 2014; Ansar et al., 2015) y posición del hueso hioides (Ferraz et al., 2007; Chaves et al., 2010), entre otros.

De la misma forma, la influencia de la función respiratoria en el desarrollo de estructuras orofaciales y postura craneocervical ha sido ampliamente discutida. Estudios previos reportan modificaciones en la postura cráneocervical relacionadas con obstrucción de la vía aérea/respiración oral
(Lima et al., 2004; Muto et al., 2002; Ucar et al., 2012; Sonnesen et al., 2017), así como estrecha relación entre la ubicación del hueso hioides (Janicka \& Halczy-Kowalik, 2006; Chaves et al.) y el tamaño de la vía aérea faríngea (Yamaoka et al., 2003), sin embargo, la asociación entre los trastornos posturales y el modo respiratorio oral no ha sido esclarecida completamente. Investigaciones sostienen que la población pediátrica con modo respiratorio oral puede desarrollar modificaciones en la postura craneocervical; no obstante, otros estudios no concluyen esta relación (Neiva et al., 2009; Bolzan et al., 2011).

La obstrucción nasal conduce a modificaciones en el patrón respiratorio, que conllevan la presencia de respiración oronasal u oral, la que clínicamente se ha asociado con alteraciones posicionales del esqueleto facial, maloclusión dentaria, alteraciones nutricionales y el bajo rendimiento académico en sujetos pediátricos y adultos. A su vez, la respiración con predominio oral conduce a adaptaciones posturales

\footnotetext{
${ }^{1}$ Facultad de Ciencias de la Salud, Universidad Autónoma de Chile, Temuco, Chile.

${ }^{2}$ Doctorado en Ciencias Morfológicas, Facultad de Medicina, Universidad de La Frontera, Temuco, Chile.

${ }^{3}$ División de Cirugía Oral, Facial y Maxilofacial, Universidad de La Frontera, Temuco, Chile.

${ }^{4}$ Centro de Excelencia en Estudios Morfológicos y Quirúrgicos (CEMyQ), Universidad de La Frontera, Temuco, Chile.
} 
para compensar la disminución del flujo de aire nasal y permitir la respiración (Josell, 1995), que gatillan modificaciones en la posición mandibular y lingual, que podrían asociarse a tonicidad muscular orofacial reducida (Valera et al., 2003) y trastornos miofuncionales orofaciales como alteración funcional de la deglución y/o trastornos de los sonidos del habla, los cuales poseen alta prevalencia en la clínica fonoaudiológica.

El objetivo del estudio fue evaluar parámetros cefalométricos de la región craneocervical, hioidea y faríngea superior, comparando sujetos adultos con respiración oral (RO) y respiración nasal $(\mathrm{RN})$.

\section{MATERIAL Y METODO}

Se diseñó un estudio descriptivo observacional de casos y controles en 40 sujetos, sin distinción de sexo. Los sujetos incluidos fueron estudiantes universitarios con edades de entre 18 y 27 años. Todos los sujetos incluidos fueron valorados inicialmente por un observador calibrado, para determinación del modo respiratorio mediante el ítem de Valoración Funcional, empleando una Placa Oronasal Adaptada (POA), de acuerdo con el Protocolo de Evaluación Fonoaudiológica de la Respiración con Puntuación PEFORP (Susanibar \& Dacillo, 2014).

Se excluyeron los sujetos con tratamiento ortodóncico, kinesiológico de la región cráneocervical o fonoaudiológico previo, así como mujeres en período de gestación, lactancia, sujetos con malformaciones faciales, sujetos antecedentes de trauma o patología quirúrgica del área maxilo facial y cervical. Los sujetos incluidos eran autodeterminados en normalidad facial y no se realizó ningun tipo de análisis de deformidad dentofacial.

Protocolo de evaluación y obtención de imagen. Los sujetos se dividieron en dos grupos, de acuerdo al diagnóstico clínico del modo respiratorio: $\mathrm{RO}(\mathrm{n}=20)$ y $\mathrm{RN}(\mathrm{n}=20)$. En cada sujeto fue obtenida una imagen radiográfica bidimensional lateral de cráneo y columna cervical, para realizar análisis cefalométrico según el protocolo descrito por Rocabado (1984) y valoración de la curvatura cervical a través de la técnica de Penning (Penning, 1968).

La telerradiografía fue estandarizada en la posición habitual de cabeza, ubicando al paciente de pie durante la toma, observando hacia el horizonte, descalzo, con separación de $10 \mathrm{~cm}$ entre ambos tobillos, sosteniendo en cada mano $2 \mathrm{~kg}$ de peso, cuidando la posición de hombros con relación a la región cervical, para permitir el estudio craneocervical completo. Se solicitó realizar dos ciclos respiratorios mediante inspiración profunda, seguida de una espiración suave para conseguir la posición de reposo habitual; posteriormente el sujeto realizó apnea para evitar la movilidad faríngea durante la captura de imagen. La captura de imagen se realizó en el equipo KXO-30R (Toshiba) y el análisis de las mismas se efectuó mediante el digitalizador y sistema de imágenes CARESTREAM DirectView Vita CR. No se utilizó cefalostato.
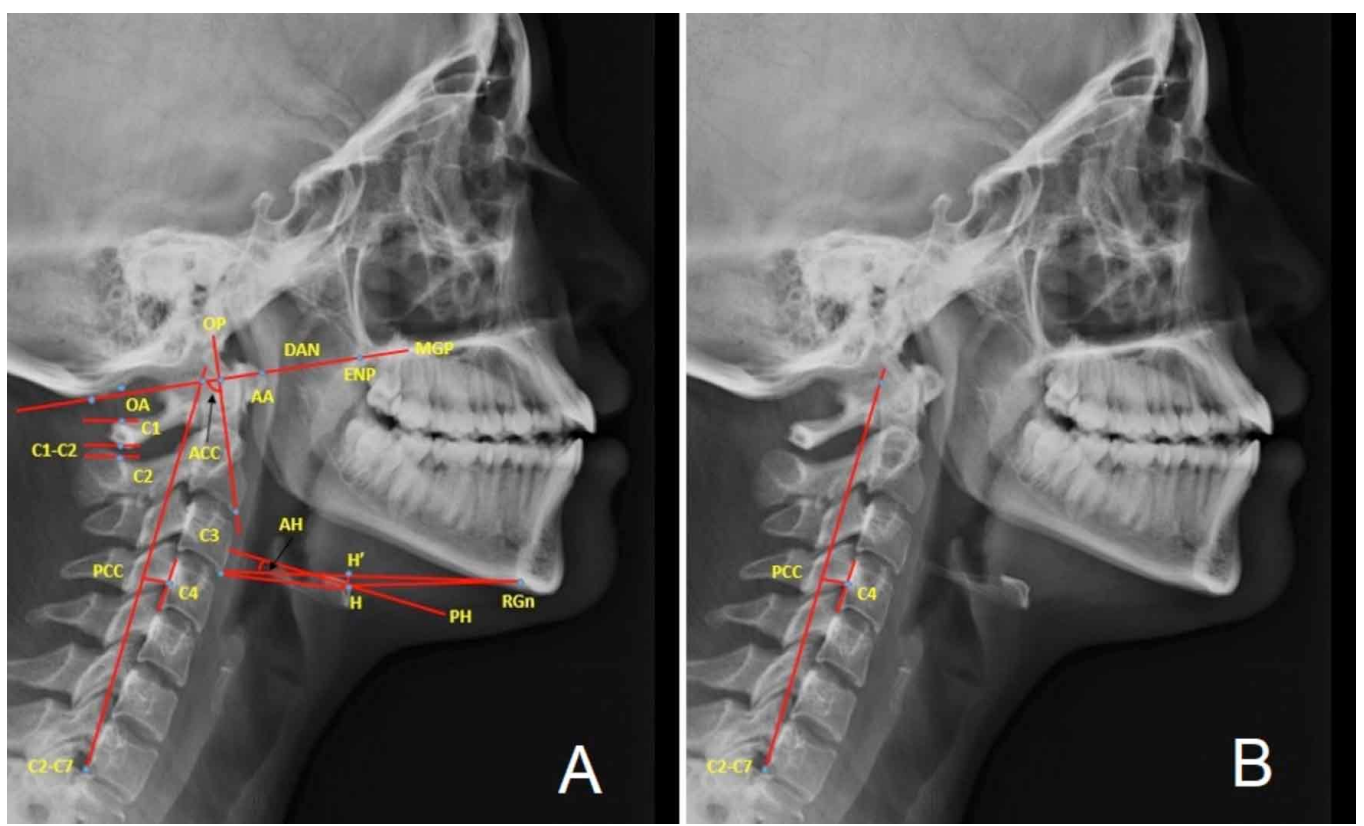

Fig. 1. Análisis cefalométrico craneocervical. A) Análisis cefalométrico craneocervical y del triángulo hioideo, según técnica de Rocabado. B) Medición de la profundidad de la columna cervical, según técnica de Penning. 
Todas las mediciones fueron realizadas de acuerdo al protocolo de Rocabado y Penning (Fig. 1) por un observador en dos tiempos diferentes con intervalo de 15 días entre ellos utilizando 10 imágenes; para ello se calculó el coeficiente de correlación intraclase (ICC), el que fue mayor a 0,9 en todas las mediciones realizadas, indicando mediciones altamente confiables. En Tabla I se encuentran las mediciones realizadas en términos de parámetros y definiciones empleados en el análisis cefalométrico.

Análisis Estadístico. Todos los datos fueron registrados en milímetros, exceptuando ACC y AH, los que fueron registrados en grados. Para el análisis estadístico se utilizó el software IBM SPSS versión 23, realizando un análisis exploratorio para conocer la distribución de las variables en estudio, aplicándose la prueba de Shapiro-Wilk, según el tipo de respiración. Posteriormente, para la comprobación de hipótesis, se aplicó la prueba T de Student para muestras independientes en las variables con distribución normal y U de Mann-Whitney en las variables con distribución no normal. Las hipótesis fueron comprobadas considerando un valor de $\mathrm{p}<0,05$.

Tabla I. Parámetros y definiciones de cefalometría craneocervical.

MGP (Plano de McGregor): trazo que va desde la ENP a la base del occipital.

OP (Plano odontoideo): línea que une el margen anteroinferior del cuerpo del axis y el ápice del proceso odontoides.

ACC (Ángulo craneocervical): ángu lo formado por la intersección del MGP y OP. Valor de referencia $101 \pm 5^{\circ}$.

OA (Distancia C0-C1): distancia entre la base del hueso occipital y el arco posterior del atlas. Valor de referencia $6,5 \pm 2,5 \mathrm{~mm}$

C1 (Atlas): primera vértebra de la columna cervical

C2 (Axis): segunda vértebra de la columna cervical

C3: tercera vértebra de la columna cervical

C4: cuarta vértebra de la columna cervical

Distancia C1-C2: distancia entre el arco posterior del atlas y el proceso espinoso de C2. Valor de referencia $6,5 \pm 2,5 \mathrm{~mm}$.

Línea C2-C7: línea entre el margen posterosuperior del ápice del proceso odontoides y el punto más posteroinferior del cuerpo de C7.

PCC (Profundidad columna cervical): des de línea C2-C7, trazar línea perpendicular hasta la parte más profunda del cuerpo de C4. Valores de referencia: cifosis cervical $<1 \mathrm{~mm}$ (valor negativo si es $<0 \mathrm{~mm}$ ), rectificación cervical $<8 \mathrm{~mm}$, valor normal curva fisiológica 8 a $12 \mathrm{~mm}$, hiperlordosis cervical $>12 \mathrm{~mm}$.

AA (Arco anterior del atlas): punto más anterior del cuerpo del atlas.

ENP(Espina nasalposterior): pun to más posterior del paladar duro.

DAN (Dimensión anterior de la nasofaringe): distancia entre AA y ENP. Valor de referencia 32,9 $\pm 3,7 \mathrm{~mm}$.

H (Hyoidale): pun to más anterior y superior del cuerpo del hueso hioides.

H': distancia perpendicular entre el punto H y la línea C3-RGn. Valor de referencia $5 \pm 2 \mathrm{~mm}$.

RGn (Retrognation): punto más posterior e inferior de la sínfisis mandibular.

Línea C3-H: línea entre el punto más anterior e inferior de C3 al punto más superior del cuerpo del hueso hioides.

Línea H-RGn: línea entre el punto más superior del cuerpo del hueso hioides al punto más posterior e inferior de la sínfisis mandibular.

Línea C3-Rgn: línea entre el punto más anterior e inferior de $\mathrm{C} 3$ al puntomás posterior e inferior de la sínfisis mandibular. PH (Plano hioid eo): plano formado desde Hy la tangente a los cuernos posteriores del hueso hioides.

AH (Ángulo hioideo): ángulo más posterior y superior formado por la intersección del PH con la línea C3-RGn. Valor de referencia $25,63 \pm 10,59^{\circ}$.

Triángulo hioideo: triángulo formado por la unión de los puntos C3,H y RGn. Valores de referencia: línea C3-RGn: 67,20土6,6

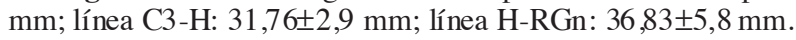

\section{RESULTADOS}

Los sujetos incluidos presentaban una eded de entre 18 y 27 años (Promedio de 21,55 años) y correspondieron a 30 sujetos del sexo femenino y 10 sujetos del sexo masculino. Se distribuyeron con normalidad las variables ACC, OA, AH, C3-RGn, C3-H y DAN. No existió distribución normal para las variables: $\mathrm{C} 1-\mathrm{C} 2, \mathrm{PCC}, \mathrm{H}-\mathrm{H}^{\prime}$ y H-RGn.

Es posible identificar que el valor promedio de la distancia OA es menor en sujetos con respiración oral, exis- tiendo diferencia significativa al comparar este parámetro en sujetos con respiración nasal ( $\mathrm{p}=0,036)$; no existió diferencia estadísticamente significativa entre los restantes valores cefalométricos estudiados al comparar sujetos de ambos grupos (Tabla II).

No obstante, respecto de los valores cefalométricos de la región craneocervical, los valores promedio son numéricamente menores para el ACC, para OA y la DAN, aun- 
que sin diferencias estadísticamente significativas. La mediana para la distancia $\mathrm{C} 1-\mathrm{C} 2$ es numéricamente menor en sujetos con respiración oral, con rangos entre 1,4 y 17,9 mm. La mediana para la PCC es numéricamente mayor en sujetos con respiración oral, con rangos entre $-1,2$ y $24,8 \mathrm{~mm}$.

Respecto de los valores cefalométricos hioideos, la mediana para H-H' es numéricamente menor en sujetos con respiración oral, con rangos entre 0,0 y $11,3 \mathrm{~mm}$. La mediana para $\mathrm{H}-\mathrm{RGn}$ es mayor en sujetos con respiración oral, con rangos entre 25,2 y $61,9 \mathrm{~mm}$. El valor promedio es numéricamente mayor para $\mathrm{C} 3-\mathrm{H}$ en sujetos con respiración oral y numéricamente menor para el $\mathrm{AH}$. El valor promedio para C3-RGn no presentó variaciones entre los grupos de estudio.

Tabla II. Comparación de parámetros cefalométricos según grupos de estudio.

\begin{tabular}{lccc}
\hline $\begin{array}{l}\text { Parámetros } \\
\text { Cefalométricos }\end{array}$ & $\begin{array}{c}\mathrm{RO} \\
(\mathrm{n}=20)\end{array}$ & $\begin{array}{c}\mathrm{RN} \\
(\mathrm{n}=20)\end{array}$ & $\begin{array}{c}\text { Estadístico } \\
\text { de prueba }\end{array}$ \\
\hline ACC $\left(^{\circ}\right)$ & $94,43+7,06$ & $98,20+7,09$ & $0,101^{\mathrm{a}}$ \\
$\mathrm{OA}(\mathrm{mm})$ & $6,29+4,12$ & $9,07+3,93$ & $0,036 \mathrm{a}^{*}$ \\
$\mathrm{AH}\left({ }^{\circ}\right)$ & $8,20+10,38$ & $10,33+14,00$ & $0,589^{\mathrm{a}}$ \\
C3-RGn (mm) & $90,64 \pm 11,44$ & $90,69 \pm 9,53$ & $0,988^{\mathrm{a}}$ \\
C3-H $(\mathrm{mm})$ & $43,74+5,49$ & $42,71+3,41$ & $0,481^{\mathrm{a}}$ \\
D AN $(\mathrm{mm})$ & $31,11+5,57$ & $32,39+6,24$ & $0,498^{\mathrm{a}}$ \\
$\mathrm{C} 1-\mathrm{C} 2(\mathrm{~mm})$ & $4,65(1,4-17,9)$ & $5,30(3,5-8,7)$ & $0,273^{\mathrm{b}}$ \\
PCC $(\mathrm{mm})$ & $6,85(-1,2-24,8)$ & $4,20(0,0-19,7)$ & $0,507 \mathrm{~b}$ \\
H-H' $(\mathrm{mm})$ & $3,70(0,0-11,3)$ & $4,80(0,0-16,6)$ & $0,607 \mathrm{~b}$ \\
H-RGn $(\mathrm{mm})$ & $48,45(25,2-61,9)$ & $46,0(36,8-68,8)$ & $0,818 \mathrm{~b}$ \\
\hline
\end{tabular}

RO = Respiración Oral; RN= Respiración Nasal *Se consideró p $<0,05$ como umbral para una diferencia estadísticamente significativa. Datos expresados como Media \pm D.S o mediana y valores mínimo-máximo, en caso de no normalidad. a Prueba T b U de Mann-Whitney

\section{DISCUSIÓN}

Nuestros resultados demuestran que en este grupo de estudio existen limitadas diferencias cefalométricas entre sujetos con respiración oral y con respiración nasal. Hallazgos similares han sido reportados por autores como Juliano et al. (2009) y D'Ascanio et al. (2010), aunque estos fueron realizados en población infantil que presentan diferencias morfologicas y funcionales importantes respecto del adulto.

La distancia OA fue el único valor que presentó diferencias estadísticamente significativas entre los grupos de estudio, siendo más reducida en sujetos con respiración oral. El valor del ACC en respiradores orales también se presentó reducido, con una media de $94,43^{\circ}$. De acuerdo con Rocabado, ángulos menores a $96^{\circ}$ implicarán rotación posterior aumentada de cráneo que se vincula con la disminución del espacio OA. Este mismo autor sostiene que, el valor reducido de OA también se relaciona con un aumento en la distancia C3RGn, lo cual no fue evidenciado en nuestro análisis.
Además, fue evidenciada una distancia C3$\mathrm{H}$ por sobre la norma para ambos grupos de estudio, lo cual indica posición anteriorizada del hueso hioides. El aumento en la tensión muscular de los músculos suprahioideos, que tienen inserción mandibular (vientre anterior del M. digástrico, $\mathrm{M}$. milohioideo y M. genihioideo), son responsables importantes en esta condición, toda vez que Yamaoka et al., refirieron que los músculos que tienen inserción en el hueso hioides juegan un papel importante en la mantención de una vía aérea normal, observando que la ubicación del hueso hioides también podría modificarse con diferentes posiciones mandibulares. Resultados similares fueron reportados por Juliano et al. tras evaluar sujetos con respiración oral.

Los hallazgos evidencian medidas de $\mathrm{AH}$ que no difieren estadísticamente entre los grupos de estudio, presentándose en ambos casos un valor menor a los valores de normalidad establecidos por Bibby \& Preston (1981) para este parámetro, lo cual sugiere descenso y/o con rotación inferior del hueso hioides, lo cual podría estar siendo influenciado por la musculatura infrahioidea y, especialmente, por músculos con inserción en los cuernos mayores del hueso hioides, tal como los constrictores de la faringe.

A pesar de estas asociaciones, el posicionamiento del hueso hioides y su rol en normalidad sigue siendo controversial, ya que Yamaoka et al. plantearon que el hueso hioides mantiene una posición estable con la finalidad de asegurar proporciones adecuadas en las vías respiratorias, siendo independiente del patrón respiratorio; por otra parte, la posición de la mandíbula presenta relación positiva con la posición del hueso hioides, donde la protrusión mandibular se caracteriza por la posición adelantada del hueso hioides con mayor tamaño de vía aérea y la posición retruída de la mandíbula genera un efecto inverso (Olate $e t$ al., 2014); en base a estos hallazgos es probable que la condición respiratoria oral o nasal sea una variable de menor influencia en la posición del hueso hioides en comparación con la variable posición de la mandíbula dentro del contexto craneofacial.

Nuestro estudio no asoció las modalidades de respiración estudiadas y el espacio aéreo nasofaríngeo o superior, contrario a lo reportado por otros estudios, en los cuales se señala la pre- 
sencia de espacio nasofaríngeo menor en sujetos con respiración oral (Juliano et al.; Chung Leng Muñoz \& Beltri Orta). En este escenario, la ausencia de diferencias observadas entre nuestros grupos de estudio pueden relacionarse con: 1) composición muestral y número muestral insuficiente para obtener relaciones significativas, 2) errores en el método de diagnóstico del modo respiratorio que divide a los sujetos en ambos grupos (RO y RN), 3) errores en el método de evaluación cefalométrica que limita el análisis de diferencia y se centra en la comparación de medidas que pueden no ser relevantes para diferenciar ambos grupos o 4) que definitivamente la modalidad de respiración oral o nasal sea vinculada principalmente a la condición esqueletal de posicionamiento maxilo-mandibular y sea esta condición morfológica más relevante que el hábito respiratorio generado en el sujeto.

Bajo las limitaciones de esta investigación, podemos concluir que no existen diferencias significativas en el tamaño sagital de vía aérea superior y valores cefalométricos de la región craneocervical e hioidea entre sujetos adultos con modo respiratorio nasal y oral; en futuras investigaciones deben ser consideradas las condiciones de morfología facial o maxilo-mandibular, para determinar más adecuadamente la influencia de los parámetros cefalométricos en el diagnóstico del modo respiratorio.

\section{AGRADECIMIENTOS}

Los autores María P. Moya y Juan P. Baeza agradecen a la Universidad Autónoma de Chile por financiar este estudio a través del Fondo de Investigación DIP99-16 y a la Dra. Tamara Otzen por su guía metodológica en el desarrollo del presente trabajo.

MOYA, M. P.; OLATE, S. \& BAEZA, J. P. Craneocervical Analysis in Subjects With Oral and Nasal Breathing. Int. J. Morphol., 37(2):724-729, 2019

SUMMARY: The influence of respiratory function on the development of orofacial structures and craniocervical posture has been widely discussed. The objective of the study was to compare cephalometric values of the craniocervical and hyoid region in subjects with nasal and oral respiration. Subjects between 18 and 27 years of age, of both sexes, were included, where 20 presented oral breathing diagnosis and 20 did not present this alteration; using lateral telerradiography of the head and neck, craniocervical cephalometric analysis was performed of Rocabado and Penning technique was applied, obtaining craniocervical and hyoid measurements, anterior nasopharyngeal dimension and cervical curvature. For the statistical analysis we used the Shapiro-Wilk normality test and the $\mathrm{T}$ test for independent samples, considering a value of $\mathrm{p}<0.05$ to obtain significant differences; in those parameters where no normal distribution was presented, the MannWhitney U test was applied. No significant differences were found between the study groups and the cephalometric values??analyzed, except for the distance between the base of the occipital bone and the posterior arch of the atlas $(p=0.03)$. There are limited cephalometric differences between subjects with oral breathing and nasal breathing, with no association of the nasopharyngeal air space with the breathing modalities studied. Conditions of facial or mandibular morphology should be considered in order to determine more adequately the influence of cephalometric parameters in the diagnosis of the respiratory mode in future studies.

KEY WORDS: Breathing, Nasal obstruction, Cephalometry, Posture, Hyoid bone.

\section{REFERENCIAS BIBLIOGRÁFICAS}

Ansar, J.; Singh, R. K.; Bhattacharya, P.; Agarwal, D. K.; Verma, S. K. \& Maheshwari, S. Cephalometric evaluation of the airway dimensions in subjects with different growth patterns. J. Orthod. Res., 3(2):108-12, 2015.

Bibby, R. E. \& Preston, C. B. The hyoid triangle. Am. J. Orthod., 80(1):927,1981 .

Bolzan, G. de P.; Souza, J. A.; Boton, L. de M.; Silva, A. M. \& Corrêa, E. C. Facial type and head posture of nasal and mouth-breathing children. J. Soc. Bras. Fonoaudiol., 23(4):315-20, 2011.

Chaves, T. C.; de Andrade e Silva, T. S.; Monteiro, S. A.; Watanabe, P. C.; Oliveira, A. S. \& Grossi, D. B. Craniocervical posture and hyoid bone position in children with mild and moderate asthma and mouth breathing. Int. J. Pediatr. Otorhinolaryngol., 74(9):1021-7, 2010.

Chung Leng Muñoz, I. \& Beltri Orta, P. Comparison of cephalometric patterns in mouth breathing and nose breathing children. Int. J. Pediatr. Otorhinolaryngol., 78(7):1167-72, 2014.

D’Ascanio, L.; Lancione, C.; Pompa, G.; Rebuffini, E.; Mansi, N. \& Manzini, M. Craniofacial growth in children with nasal septum deviation: a cephalometric comparative study. Int. J. Pediatr. Otorhinolaryngol., 74(10):1180-3, 2010.

Ferraz, M. J.; Nouer, D. F.; Teixeira, J. R. \& Bérzin, F. Cephalometric assessment of the hyoid bone position in oral breathing children. Braz. J. Otorhinolaryngol., 73(1):45-50, 2007.

Inoko, Y. \& Morita, O. Influence of oral appliances on craniocervical posture in obstructive sleep apnea-hypopnea syndrome patients. J. Prosthodont. Res., 53(3):107-10, 2009

Janicka, A. \& Halczy-Kowalik, L. Hyoid bone position and tongue size and patency of upper airway structures. Ann. Acad. Med. Stetin., 52 Suppl. 3:53-9, 2006.

Josell, S. D. Habits affecting dental and maxillofacial growth and development. Dent. Clin. North Am., 39(4):861-70, 1995.

Juliano, M. L.; Machado, M. A.; Carvalho, L. B.; Prado L. B. \& do Prado, G. F. Mouth breathing children have cephalometric patterns similar to those of adult patients with obstructive sleep apnea syndrome. Arq. Neuropsiquiatr., 67(3B):860-5, 2009.

Lee, W. Y., Okeson, J. P. \& Lindroth, J. The relationship between forward head posture and temporomandibular disorders. J. Orofac. Pain, 9(2):161-7, 1995.

Lima, L. C.; Baraúna, M. A.; Sologurem, M. J.; Canto, R. S. \& Gastaldi, A. C. Postural alterations in children with mouth breathing assessed by computerized biophotogrammetry. J. Appl. Oral Sci., 12(3):232-7, 2004. 
Matheus, R. A.; Ramos-Perez, F. M.; Menezes, A. V.; Ambrosano, G. M.; Haiter-Neto, F.; Bóscolo, F. N. \& de Almeida, S. M. The relationship between temporomandibular dysfunction and head and cervical posture. J. Appl. Oral Sci., 17(3):204-8, 2009.

Muto, T.; Takeda, S.; Kanazawa1, M.; Yamazaki, A.; Fujiwara, Y. \& Mizoguchi, I. The effect of head posture on the pharyngeal airway space (PAS). Int. J. Oral Maxillofac. Surg., 31:579-83, 2002.

Neiva, P. D.; Kirkwood, R. N. \& Godinho, R. Orientation and position of head posture, scapula and thoracic spine in mouth-breathing children. Int. J. Pediatr. Otorhinolaryngol., 73(2):227-36, 2009.

Olate, S.; Cantín, M.; Vásquez, B.; del Sol, M.; Henriquez-Alarcón, M. \& de Moraes, M. Pharyngeal airway space in subjects with class II and class III facial deformities. Int. J. Morphol., 32(4):1271-6, 2014.

Penning, L. Functional Pathology of the Cervical Spine: Radiographic Studies of Function and Dysfunction in Congenital Disorders, Cervical Spondylosis and Injuries. Amsterdam, Excerpta Medica Foundation, 1968.

Rocabado, M. Análisis biomecánico craneocervical a través de una telerradiografía lateral. Rev. Chil. Ortod., 1(1):42-52, 1984.

Rodríguez Romero, B.; Mesa Jiménez, J.; Paseiro Ares, G., \& González Doniz, M. L. Síndromes posturales y reeducación postural en los trastornostemporomandibulares. Rev. Iberoam. Fisioter. Kinesiol., 7(2):83-98, 2004.

Solow, B. \& Siersbaek-Nielsen, S. Cervical and craniocervical posture as predictors of craniofacial growth. Am. J. Orthod. Dentofacial Orthop., 101(5):449-58, 1992.

Sonnesen, L.; Petersson, A.; Berg, S. \& Svanholt, P. Pharyngeal airway dimensions and head posture in obstructive sleep apnea patients with and without morphological deviations in the upper cervical spine. $J$. Oral Maxillofac. Res., 8(3):e4, 2017.

Susanibar, F. \& Dacillo, C. Evaluación Fonoaudiológica de la Respiración: Protocolo de Evaluación Fonoaudiológica con Puntuación PEFORP. Lima, Ediciones Libro Amigo, 2014.

Svanholt, P.; Petri, N.; Wildschiødtz, G.; Sonnesen, L. \& Kjaer, I. Associations between craniofacial morphology, head posture, and cervical vertebral body fusions in men with sleep apnea. Am. J. Orthod. Dentofacial Orthop., 135(6):702.e1-9, 2009.

Ucar, F. I.; Ekizer, A. \& Uysal, T. Comparison of craniofacial morphology, head posture and hyoid bone position with different breathing patterns. Saudi Dent. J., 24(3-4):135-41, 2012.

Valera, F. C.; Travitzki, L. V.; Mattar, S. E.; Matsumoto, M. A.; Elias, A. M. \& Anselmo-Lima, W. T. Muscular, functional and orthodontic changes in pre school children with enlarged adenoids and tonsils. Int. J. Pediatr. Otorhinolaryngol., 67(7):761-70, 2003.
Yamaoka, M.; Furusawa, K.; Uematsu, T.; Okafuji, N.; Kayamoto, D. \& Kurihara, S. Relationship of the hyoid bone and posterior surface of the tongue in prognathism and micrognathia. J. Oral. Rehabil., 30(9):914-20, 2003.

\author{
Dirección para correspondencia: \\ Maria Paz Moya Daza \\ Facultad Ciencias Salud \\ Univerisdad Autónoma \\ Av. Alemania 01090 \\ Temuco \\ CHILE
}

Email: maria.moya@uautonoma.cl

Recibida : 04-01-2019

Aceptada: 26-02-2019 